\title{
Study on the Cultivation of Medical Students' Social Psychological Professional Skills
}

\author{
Yafan $\mathrm{Hu}$ \\ Department of Social Science \\ Qiqihar Medical University \\ Qiqihar, China 161006
}

\begin{abstract}
At present, the doctor-patient relationship is in a tense trend, many medical workers rely solely on technical apparatus and instruments for clinical diagnosis and treatment. Yet there is inadequate communication with patients, besides, current medical system is not perfect, mutual trust cannot be established between doctors and patients, furthermore, the doctor-patient relationship is commercialized gradually in the market economy, the contradiction between doctors and patients becomes more and more prominent, medical disputes have been increasing year by year. Therefore, it is an important task for the medical education reform to strengthen the education of medical students in professional spirits and cultivate the "Social Psychological Professional Skills".
\end{abstract}

Keywords—social psychological; professional skills; doctorpatient communication

\section{INYTODUCTIN}

The development of health service of our country must solve the problem on how to build a harmonious physicianpatient relationship. The tension of physician-patient relationship is caused by many reasons, including the lack of "humanities and medical practice skills" of medical staff, which is a distinctly important aspect. Most doctor training systems of our country have paid attention to the training of medical knowledge and professional skills instead of the humanistic spirit and medical practice skills. Therefore, it becomes an important task for the reform of medical education to strengthen the education on professional spirit of medical students and train the "humanities and medical practice skills". It is necessary to let the humanities and medical education become a part of the higher medical education, train the qualified talents "have both integrity and skills" and humanize the medical science and service, building a harmonious physician-patient relationship.

\section{Status Quo Of Lack Of Humanistic Medicine}

Traditionally doctors bear the sacred mission of saving lives and to fulfill the noble duty of treatment and rescue. Doctors are saviors "curing the sick". However, due to various reasons, the current doctor-patient relationship is much tense than ever, medical disputes and medical causes are reported one after another. The patients, in order to get a favorable result, often quarrel, wrangle, fight or smash in hospitals even kill doctors. Meanwhile, medical workers also feel anxious, wronged and helpless, the hospitals are unable to go as usual. Funny things happen as medical worker wearing helmets at work, public security officers invited to serve as vice president of hospitals and so on. Currently doctors and patients who should work together to fight against diseases have to fight a civil war. Main reasons include lower investment and imperfect medical security system; over higher expectations of patients to medical and technical level and treatment effect, and media hype and misleading. However, the problems of doctors cannot be ignored either. Medical workers have no active consciousness to serve patients, no mutual communication and are limited to respond to complicated situations, and the lack of practicing humanistic medicine is a main reason. Yet the lack of humanistic education on medical students in colleges is one of the sources for the result. It is mainly shown as follows:

\section{A. Centering on Professional Education and Ignoring the Humanistic Quality}

In the curriculum setting in medical schools, medical and professional courses occupy an absolutely high proportion, yet the humanistic courses, whether in credit hour or check requirements, are poorly set, standing at a marginal area. In the atmosphere, no attentions are paid whether in teaching or learning. After graduation, the graduates see more diseases than patients and examinations are conducted through instruments instead of interrogation and physical examination, and bedside intercom systems are used instead of ward visits, which cause extraordinary prescription, abused examination. The blocked communication between doctors and patients, lack of trust, and indifferent attitudes of medical workers as well as unaffordable medical expenses finally cause crisis of trust between doctors and patients.

\section{B. Centering on Learning Humanistic Theory But Ignoring the Cultivation of Humanistic Skills}

In recent years, the release of "global lowest basic requirements" for undergraduate medical education around the world and the situation of tense doctor-patient relationship at home have aroused the attentions of scholars. Many schools and colleges began to regard the medical humanities education, and set medical humanities education courses, and increase class hours. However, most are set as elective courses; the contents aim at knowledge; students are still taught in class; and the examination is still based on papers, most are still in 
idle theorizing; and it still has large gap with the clinical application. Once students are pushed to the clinical, facing patients, they will feel helpless in the actual problems, and they have to learn and accumulate experience in lessons.

\section{Focusing on Improving Individual Abilities, Ignoring the Team Spirit}

The medical work needs mutual cooperation between doctors, medical workers, medical technicians, doctors and patients, and the teamwork is a must to rely on to ensure efficient diagnosis and treatment. Within the team, doctors are generally organizers and leaders, who must propose decisionmaking programs and countermeasures in time. If the doctors have no senses of teamwork, cooperation and leadership quality, the work will be hard to be well done. For a long time, facing the examination-oriented education pattern, teaching methods, few group discussions, extracurricular study in learning in China, most students are dedicated to learning instead of cooperation. In addition, with the students increased in enrollment after the increased enrollment, the faculties are inadequate, due to higher medical requirements of patients and restrictions in laws and regulations, teaching cases are difficult to find and so on, organizing groups to learn and bedside discussion are also difficult. Actually the learning patterns will help students develop a sense of teamwork, organizational abilities and spirits of cooperation and win-win.

\section{It Is AN URGENT NEEd For MedicAl DEVELOPMENT TO STRENGTHEN THE CUlTIVATION OF MEDiCAL STUdENTS PROFESSIONAL SKILLS IN HUMANISTIC MEDICINE}

The object of medical research shall be a person, who has not only natural attributes, but also social attributes. The social attributes of a person determine that doctors shall not only know the health and disease, but also understand patients and the society, correctly master the disease-person-society relations, and be adapted to the transfer from biomedical to bio-psycho-social medicine, and make sure of medical humanity, human culture. Medicine is a complex social systematic engineering and the medical and health activities are not limited to a narrow range of profession, and it has close relations with the society, politics, economy, culture, and doctors must master knowledge related to social medicine, health economics, medical psychology, health management, health laws and regulations and the like. Therefore, the humanistic spirit should be promoted in medicine, strengthen the medical humanities education and realize the co-existence and blending of medicine and humanities. Social Psychological Professional Skills" mainly include scientific values, professional service attitude, ability to communicate with patients, basic legal quality, medical work management, medical team spirit of cooperation and psychological adaptability. The capabilities are involved in not only the development of doctors, but also the treatment effect of patients and the hospital construction and development. To strengthen the humanities education has become a strong international trend of medical education. In the 1980s, the America Continuing Medical Education (CME) made it clear that it was necessary to strengthen the social psychological professional education of medical students, and pointed out,
"Doctors who have no social psychological professional basis often lose capabilities in intellectual challenges and response to the challenges. In 1993, the British General Medical Committee (GMC) proposed that more humanities learning modules should be added to the medical education and practice in order to achieve the mutual penetration between medical humanities and medical natural science and inclusive; the Institute for International Medical Education (IIME), founded in 1999, established the International Minimum Essential Requirements for undergraduate medical education, and stressed that dedication to work and ethic behaviors are cores of medical practice, and professional values, attitudes, behavior and ethic, should be taken as basic requirements the same as medical knowledge and clinical skills, which is one of the core capabilities and a basic quality. All of these are requirements of contemporary health development and medical education, and also propose new goals for the medical humanities education. In order to make up for the deficiency of humane quality in most physicians and improve the Social Psychological Professional Skills of physicians, the Chinese Medical Doctor Association invited experts at home and abroad for a two-year survey and research and compiled the first textbook of Social Psychological Professional Skills for Physicians in the country and launched the training and examination of Social psychological professional skills for physicians across China. Considering that the "Social Psychological Professional Skills" are much regarded, which may even become compulsory contents for doctors to obtain medical qualification, therefore, medical schools and colleges should fully understand the importance and urgency to strengthen the social psychological professional skills of medical students, deepen the educational reform and effectively strengthen the humanities medical education. The talents trained shall not only have medical professional knowledge and ability, but also own solid social psychological professional skills.

\section{Measures Taken To Strengthen The Cultivation OF MEDICAL STUDENTS' SOCIAL PSYCHOLOGICAL PROFESSIONAL SKILLS}

Social Psychological Professional Skills play an important role in the career of doctors, famous medical experts have their achievements reflected not only in their professional quality, but also in their noble humanistic quality and noble professional ethics. Therefore, acting as physicians in the future, medical students shall not only develop professional knowledge and skills in schooling stage but also own humanities and medical knowledge and professional skills in order to become qualified talents.

\section{A. Deepen the System Reform and Strengthen the} Construction of Medical Humanities Curriculum

Humanities curriculum is an important part of medical education. Through the learning of medical humanities knowledge, students can deeply understand the connotation of the doctors-patient relationship, and embody the nature of "Medicine is an art of benevolent" in practice in the future. In the curriculum setting, regard the integral construction and the construction of core courses, break the inherent boundaries of 
courses, strengthen the integration of courses, enrich students' humanities through various forms, and make the medical humanities course go throughout the whole medical education, so that students, while learning professional knowledge, are aware of the importance of humanities courses in medicine, and consciously strengthen the improvement of humanistic quality of their own.

1) Reform teaching plans: In order to add the humanities courses, first of all, add humanistic medical course, and courses as Medical Ethic, Medical Psychology, and Health Law and so on are set in many medical schools and colleges. Aiming at the actual needs of current clinical work facing the "difficult to see a doctor, expensive for treatment" and the tense doctors-patient relationship, it is necessary to set Doctors-Patient Communication, Health Economics, etc., and foster students' concepts in skills communication and cost control. The establishment of humanities curriculum shall not only to enable students to master theoretical knowledge of humanities medicine, but also make them master the practice of humanities medicine and master the practical skills in clinical practice. Second, it is necessary to reform the teaching contents of humanities courses, teaching methods, and change the previous theoretical courses, add teaching practices, link the clinical work to practice, and carry out the case teaching, focus on experiential and situational teaching, make students understand the current status quo of doctor-patient relationship, and actively explore ways and methods to improve it, and take the initiative to participate in humanities teaching reform, broaden the horizons of theory, train the practice ability and improve the quality of the humanities.

2) Promote the integration of profession and humanities, and cultivate the professional quality of medical students and make the humanities content go through professional teaching: The integration of professional knowledge and humanistic spirit, professional skills and humanities skills are an inevitable trend to train qualified medical talents, and a requirement to promote the medical development and achieve the social harmony. During the professional teaching, the professionalism that doctors own shall be imparted to students to foster their senses of professional sacredness and glory so as to form the professional quality of "Regard life, Care for Patients" and follow the idea "Ethic is the essence for doctors" to shape a "White Angel" image.

3) Strengthen the education of laws and regulations, form the awareness of the practicing under laws: With the improvement of medical laws, the awareness of patients to protect their rights and interest is also growing, the law has become an important criterion for coordinating the doctorpatient relationship. Medical workers must master relevant laws and regulations, and coordinate the doctor-patient relationship according to law. Therefore, it is imperative to set courses of medical laws and regulations in professional teaching, through learning the laws and regulations, medical students shall form consciousness of practicing according to laws, abide by laws and regulations, and protect the legal rights and interest of patients and that of their own through law knowledge.
4) Train communication skills and promote the doctorpatient harmony: Analyze the causes of disputes, the medical and technical defects account for less than $20 \%, 80 \%$ or more are caused by lack of communication between doctors and patients. During the training of medical students, the teaching of communication with patients, especially the teaching practice is in a serious shortage, which causes the fear of students at clinic work to communicate with patients, the communication is not clear, the communication content, ways and methods are far to satisfy patients. To communicate with patients is an important part of humanistic medicine. First, make students learn to use smiles, eyes, body, gestures, scenarios and so for non-verbal communication with patients. Second, learn oral communication with patients from communication contents, ways and methods. Third, make students learn to deal with emergency and inform bad news. Fourth, make patients participate in the diagnosis and treatment plans. The key is to enable students to grasp skills for communication, provide patients with humanistic services, both doctors and patients are united to fight against the diseases.

\section{B. Regard the Extracurricular Implicit Education outside Campus and Improve the Influence of Campus Culture}

Campus culture has great attraction and potential to explore, and the colorful campus culture can help form and shape the spirits of medical humanities and the university. During the professional education, colleges and universities should carry out healthy and lively campus cultural life, full of medical humanities atmosphere, and instruct the students through the extracurricular implicit education. Make full use of cultural activities popular in medical students as medical interviews, discussions on hot issues, theme speeches and so on, which not only highlight main themes of socialist core values, but also reflect the diverse campus culture and foster students' lofty spiritual pursuit.

\section{Improve the Quality of College Teachers and Fully Exert the Guiding Role of Teachers in the Education of Medical Students' Value}

Teachers play an important role in the formation of students' personality and values. The humanistic spirit and values of professional teachers or political theory teachers or instructors will affect the students through contacts. Therefore, in order to promote the construction of values, the construction of teachers must be regarded and strengthened. To improve the quality of teachers, it is necessary to strengthen the humanities education training on professional teachers and the training of related medical knowledge on teachers who are engaged in the humanities education. Colleges and universities should exert the guiding roles of professional teachers, political theory teachers and instructors in the education of medical students' values, and combine the three teaching forces in teaching, who should bear the glorious mission of teaching, management and service, and the responsibilities for teaching should be reflected not only in the teaching of professional knowledge, but also in the correct guiding on values. 


\section{CONCLUSION:}

It is a long term and continuous process to train medical students' social psychological professional skills, higher medical colleges and universities should create a good atmosphere of humanities and medicine, establish a scientific, systematic and holistic curriculum system, and help medical students master social psychological professional skills through various teaching forms and check modes. In the coming medical work, they are expected to provide professional service full of humanities, form a harmonious doctor-patient relationship and promote the development of the health industry.

\section{REFERENCES}

[1] Xuan Qiang et al, Training of Medical Students on Social Psychological Professional Skills and its Psychological Effects to Perioperative Patients, Anhui Medical Journal, 2014

[2] Hu Liangbo et al, Status Quo of Training of Medical Students on Social Psychological Professional Skills and Countermeasures-Based on Yongchuan Hospital affiliated to Chongqing Medical University, Medicine and Philosophy, 2016

[3] Dai Zhengqing, et al, Discussion on the Cultivation of Medical Students' Medical Students on Social Psychological Professional Skills, Chinese Journal of Hospital Administration, 2012

[4] Yuan Li, et al, Training and Practice of Medical Students' Medical Students on Social Psychological Professional Skills, Soft Science of Health, 2015

[5] Zhao Bang, et al, Theoretical Thought and Practice Research on Medical Students' Medical Students on Social Psychological Professional Skills, Chinese Journal of Cardiovascular Review, 2010

[6] Guo Honghua, et al, Research on the Application of Humanities Education in Medical Students' Training of Clinical Skills, 2012. 